\title{
ON THE DETERMINATION OF PHYSICAL CONDITIONS IN THE NEBULAE
}

\author{
A. A. Boyarchuk, R.E. Gershberg, N. V. Godovnikov, and V. I. Pronik \\ (Crimean Astrophysical Observatory, U.S.S.R.)
}

Following the well-known physical theory of recombination and forbidden-line emission, we have carried out calculations which may be useful for a quantitative analysis of the observations of planetary nebulae and other emission objects (diffuse nebulae, emission details in extragalactic nebulae, symbiotic and flare stars). As the result, we have a set of four types of graphs.

In the first-type graphs the intensity and spectral distribution of continuous hydrogen plasma radiation (free-free, free-bound and two quanta transitions) are given for different $T_{\mathrm{e}}$ and $n_{\mathrm{e}}$. The Balmer and Paschen discontinuities as functions of $T_{\mathrm{e}}$ and $n_{\mathrm{e}}$ are also given.

In the second-type graphs, the ratios of auroral and nebular lines of [OIII], [N II], [NeIII], [NeV], [OII] and [SII] ions are shown. These ratios are given in the plane $\left(\log T_{\mathrm{e}}, \log n_{\mathrm{e}}\right)$ as curves of constant ratios and can be used for the determination of $n_{\mathrm{e}}$ and $T_{\mathrm{e}}$ by Seaton's curve-intersection method.

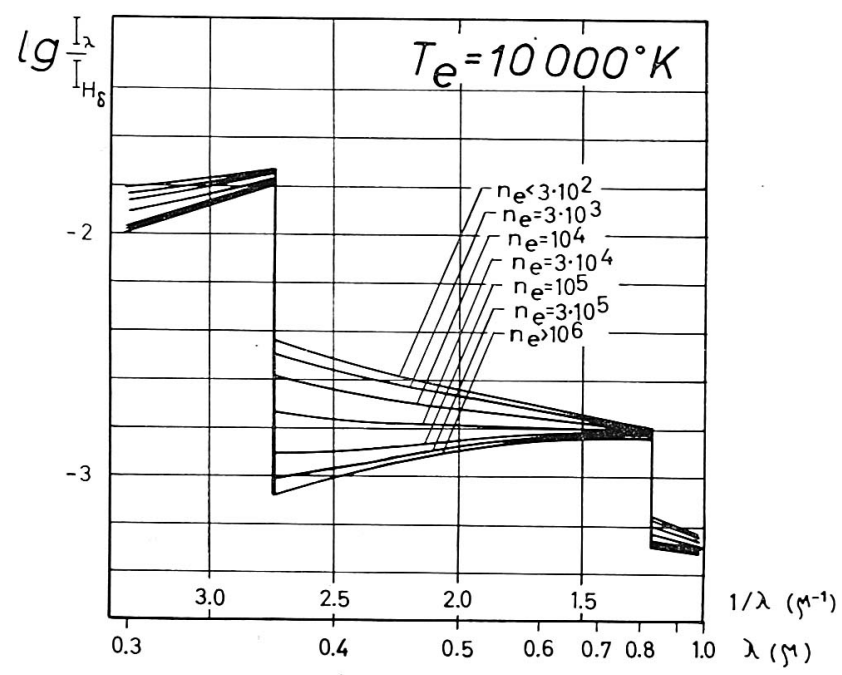

FIG. 1. Intensity distribution in continuum at $T_{\mathrm{e}}-10000^{\circ}$ as a function of $n_{\mathrm{e}}$.

Osterbrock and O'Dell (eds.), Planetary Nebulae, 162-165. O. I.U. 


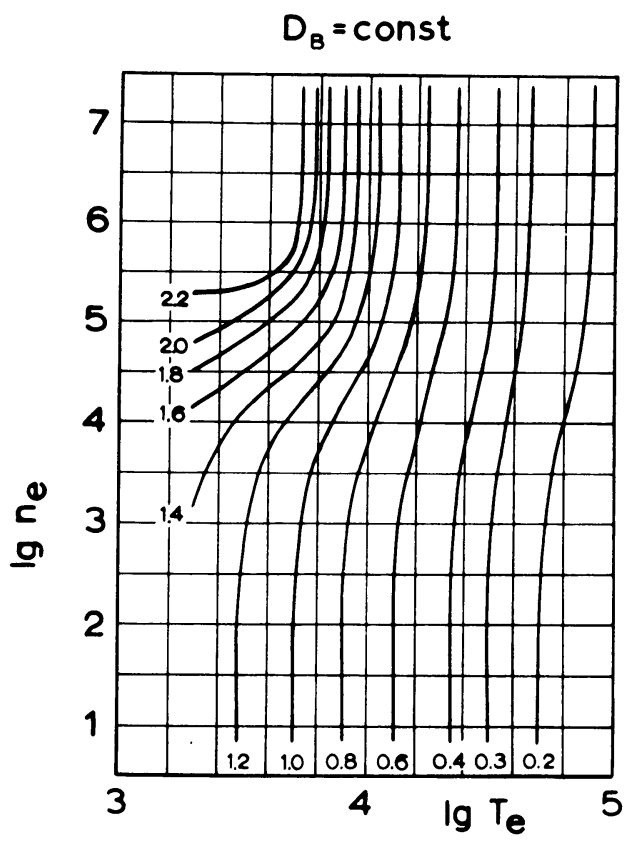

FIG. 2. Balmer discontinuity as a function of $n_{\mathrm{e}}, T_{\mathrm{e}}$.

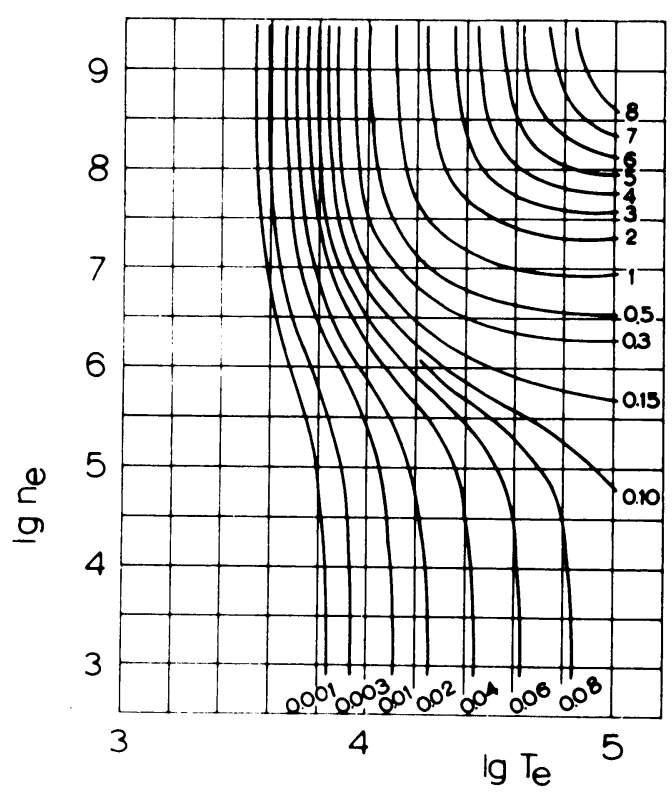

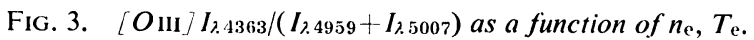


For objects optically thin in $\mathrm{H} \beta$ the intensity of a forbidden line is given by

$$
\frac{I_{i}}{I_{\mathrm{H} \beta}}={ }_{n_{\mathrm{p}}}^{n_{1}} \theta_{i}\left(n_{\mathrm{e}}, T_{\mathrm{e}}\right),
$$

where $I_{i}=$ intensity of forbidden line, $I_{\mathrm{H} \beta}=$ intensity of $\mathrm{H} \beta$ line, $n_{1}=$ the number of ions in the ground state, $n_{p}=$ the number of protons, and $\theta_{i}\left(n_{\mathrm{e}}, T_{\mathrm{e}}\right)=$ known functions of $T_{\mathrm{e}}$ and $n_{\mathrm{e}}$.

In the third-type graphs, curves of equal values of $\theta_{i}$ are given in the plane $\left(\log T_{e}, \log n_{\mathrm{e}}\right)$ for 14 lines of [OI], [OII], [OIII], [NII], [SII], [SIII], [NeIII] and [Ne v].

For stationary conditions, when $n_{1} / n_{p}$ are determined by the abundances and the Saha-Boltzmann equations for the non-equilibrium case, the ratios $I_{i} / I_{\mathrm{H} \beta}$ and the intensities $I_{i}$ can be determined as functions of $T_{\mathrm{e}}, n_{\mathrm{e}}$, temperature of radiation $T_{*}$ and dilution factor $W$. These last graphs can be used to analyze some objects with secular variations of the exciting radiation and/or other physical conditions. All these graphs will be published in Publications of Crimean Astrophysical Observatory, 38 and 39. All formulae used and references of atomic constants are given there.

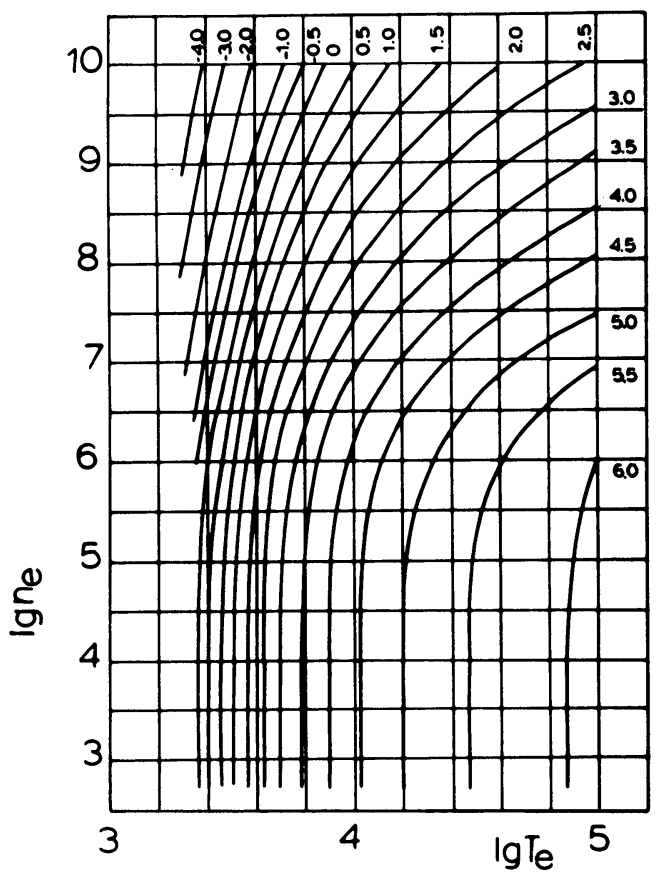

FIG. 4. $\left[O_{\mathrm{III}}\right] \lg \theta_{2.4959+\lambda .5007}$ as a function of $n_{\mathrm{e}}, T_{\mathrm{e}}$. 

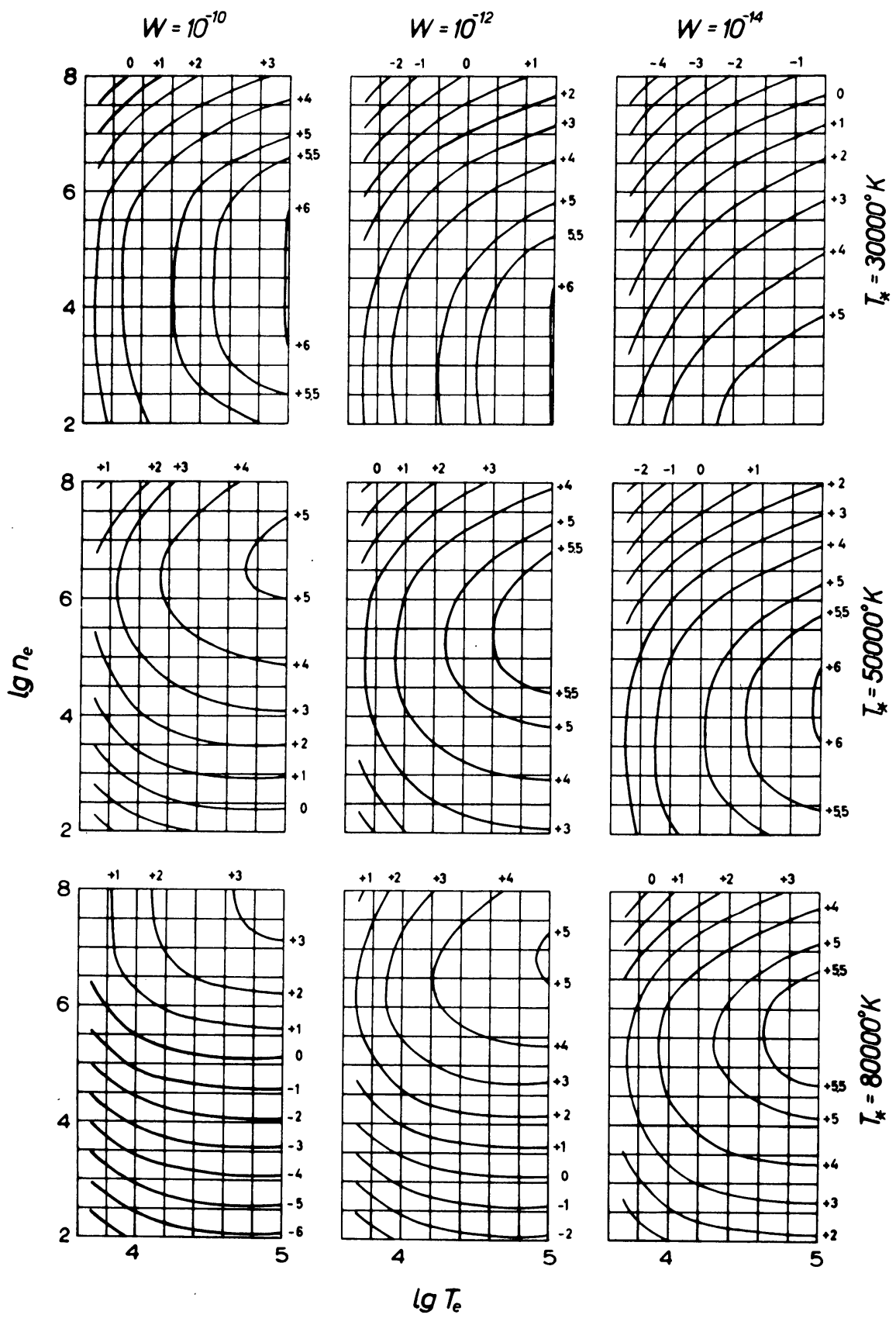

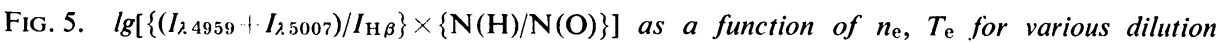
factors and various stellar temperatures $T_{*}$. 